\title{
Scanning thermoacoustic tomography in biological tissue
}

\author{
Geng Ku and Lihong V. Wang ${ }^{\mathrm{a})}$ \\ Optical Imaging Laboratory, Biomedical Engineering Program, Texas A\&M University, \\ 3120 Tamu, College Station, Texas 77843-3120
}

(Received 5 August 1999; accepted for publication 2 March 2000)

\begin{abstract}
Microwave-induced thermoacoustic tomography was explored to image biological tissue. Short microwave pulses irradiated tissue to generate acoustic waves by thermoelastic expansion. The microwave-induced thermoacoustic waves were detected with a focused ultrasonic transducer. Each time-domain signal from the ultrasonic transducer represented a one-dimensional image along the acoustic axis of the ultrasonic transducer similar to an ultrasonic A-scan. Scanning the system perpendicularly to the acoustic axis of the ultrasonic transducer would generate multi-dimensional images. Two-dimensional tomographic images of biological tissue were obtained with $3-\mathrm{GHz}$ microwaves. The axial and lateral resolutions were characterized. The time-domain piezo-electric signal from the ultrasonic transducer in response to the thermoacoustic signal was simulated theoretically, and the theoretical result agreed with the experimental result very well. (C) 2000 American Association of Physicists in Medicine. [S0094-2405(00)03105-9]
\end{abstract}

Key words: microwave, ultrasonics, thermoacoustics, photoacoustics, tomography

\section{INTRODUCTION}

Purely microwave imaging of biological tissues has been investigated for a number of years. ${ }^{1-5}$ The advantages of microwave imaging include the use of nonionizing radiation and relatively good imaging contrast. However, purely microwave imaging has had difficulties in multi-channel detection of microwave without cross coupling, in reconstruction algorithms, and especially in achieving good spatial resolution because of the large wavelength of microwaves. Purely ultrasound imaging (ultrasonography), an established medical imaging modality, can yield good spatial resolution but has poor contrast. Microwave-induced thermoacoustics may bridge the gap and combine the advantages of the two types of radiation.

In microwave-induced thermoacoustics, microwave pulses generate acoustic signals in lossy media. This phenomenon was known as microwave-auditory or microwavehearing effect in the early years. ${ }^{6}$ Microwave-induced thermoacoustics was used to quantify physical parameters in media such as the power density and the concentration of a given substance. ${ }^{7-9}$ Microwave-induced thermoacoustics was also employed by several investigators in the 1980s for imaging of biological tissues. ${ }^{10-15}$ However, these early works did not produce any tomographic or depth-resolved images. Recently, images of biological tissues were reconstructed based on microwave-induced thermoacoustics. ${ }^{16,17}$ This approach requires measurements of a large amount of data around the tissue and expensive computation following the data acquisition.

Microwave-induced thermoacoustic imaging is based on the detection of the thermoacoustic signals generated by microwaves in the samples. Pulsed microwave radiation is used to irradiate the samples. Absorbed microwave energy causes thermoelastic expansion that radiates acoustic waves. An ultrasonic transducer detects the time-resolved thermoacoustic signals. If optical radiation instead of microwave radiation is used, this phenomenon is better known as photoacoustics. Although microwave-induced thermoacoustic imaging shares similar principles with the photoacoustic imaging in the optical-wavelength region, ${ }^{18-28}$ it may have broader applications in medical imaging because microwave radiation provides a deeper penetration depth in biological tissues than light.

The electric field strength of a plane wave in a lossy media is attenuated exponentially as

$$
E=E_{0} \exp (-\alpha z),
$$

where $E_{0}$ is the electrical field at the sample surface, $E$ is the electrical field at the depth $z$, and $\alpha$ is the electric-field absorption coefficient expressed as

$$
\alpha=\omega \sqrt{\frac{\mu \epsilon}{2}\left[\sqrt{1+\left(\frac{\sigma}{\omega \epsilon}\right)^{2}}-1\right]},
$$

where $\omega$ is the angular frequency, $\mu$ is the permeability, $\epsilon$ is the permittivity, and $\sigma$ is the conductivity. The induced thermoacoustic pressure depends on the intensity of microwave and the complex dielectric constant of the material. In the frequency range of $0.1-10 \mathrm{GHz}$, the dielectric constant (ratio of the permittivity in material to that in vacuum) has a value of 5-70 for soft tissues, and the conductivity has a value of $0.02-3 \Omega^{-1} \mathrm{~m}^{-1} \cdot{ }^{29-31}$ The dielectric properties of tissues determine the absorption of microwave at various microwave frequencies. At $3 \mathrm{GHz}$, the penetration depths for fat and muscle, which are the inverse of the absorption coefficients, are $9 \mathrm{~cm}$ and $1.2 \mathrm{~cm}$, respectively; while at $500 \mathrm{MHz}$, the penetration depths for fat and muscle are $23.5 \mathrm{~cm}$ and 3.4 $\mathrm{cm}$, respectively. ${ }^{32}$ Most of the other soft tissues have an absorption coefficient in between those for muscle and fat. This wide range of values among various tissues can provide a high imaging contrast for biological tissues. Cancerous 


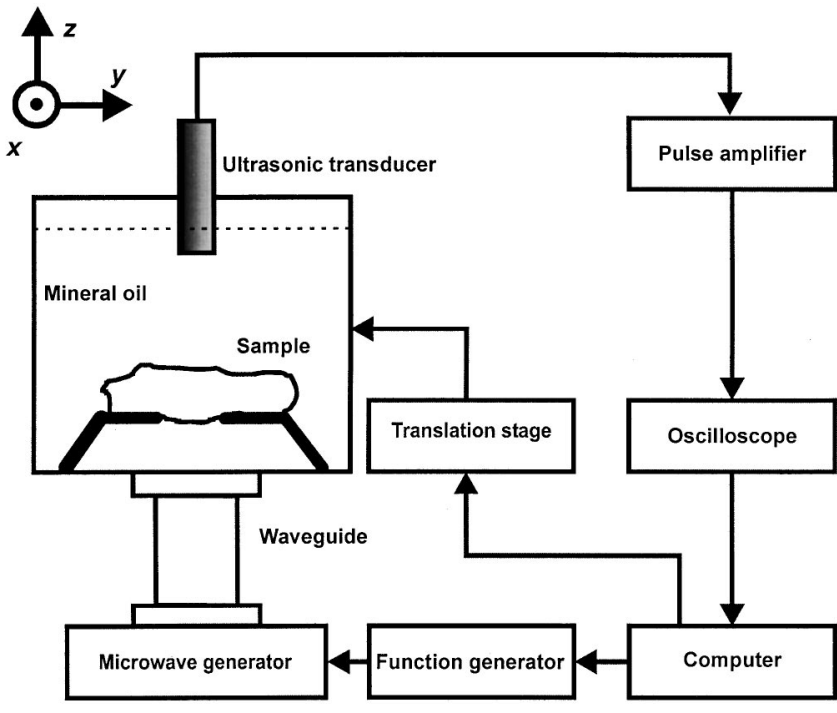

FIG. 1. Experimental setup for scanning thermoacoustic tomography.

breast tissues are $2-5$ times more strongly absorbing than surrounding normal breast tissues, which has been attributed to an increase in bound water and sodium within malignant cells. ${ }^{33-35}$ Therefore, microwave-induced thermoacoustic imaging may potentially be used to detect early-stage cancers.

We here present our studies on scanning microwaveinduced thermoacoustic tomography toward biomedical applications. The microwave-induced piezo-electric signal from the ultrasonic transducer was simulated theoretically to explain the experimental observation for the first time to our knowledge. Cross sections of biological tissues were imaged in full view using our current $3-\mathrm{GHz}$ imaging system, representing a significant advance from the previous $9-\mathrm{GHz}$ system that imaged cross sections only partially because of the limited penetrating power of the radiation at $9 \mathrm{GHz} .{ }^{36}$ Our imaging approach differs significantly from the prior arts in microwave-induced thermoacoustic imaging. Lateral resolution was achieved by use of a focused ultrasonic transducer. Axial resolution was obtained by measuring the temporal profiles of the microwave-induced acoustic signals. Depthresolved tomographic images were acquired directly without resorting to image reconstruction.

\section{METHODS}

The experimental setup used for this study is shown in Fig. 1. A Cartesian coordinate system was set up for reference. The $z$ axis was along the acoustic axis pointing upward. The $x$ axis was perpendicular to the drawing plane and pointed outward. The $y$ axis was in the drawing plane pointing to the right. A 3-GHz microwave generator transmitted microwave pulses. The peak power was estimated to be 2 $\mathrm{kW}$. The pulse width was modified to $0.5 \mu \mathrm{s}$. A function generator (DS345, Stanford Research System) was employed to trigger the microwave generator, control its pulse repetition frequency, and synchronize the oscilloscope sampling. Microwave energy was delivered by a rectangular waveguide with a cross section of $72 \mathrm{~mm} \times 34 \mathrm{~mm}$. The object to be imaged was placed on a plastic stand inside a plexiglass container filled with mineral oil. The container was fixed on a two-dimensional $x-y$ translation stage (MD2, Arrick Robotics). A personal computer controlled the two stepper motors to drive the translation stage in the $x$ and $y$ directions. Both mineral oil and plexiglass have a small absorption coefficient for microwaves. Mineral oil also provides good acoustic coupling. An ultrasonic transducer was immersed in the mineral oil facing the microwave waveguide. The transducer was connected to a pulse amplifier. The amplified signal was recorded and averaged 100 times by an oscilloscope (TDS640A, Tektronix) and then transferred to a personal computer for imaging.

Two ultrasonic transducers were used in this study. For the first one (V314, Panametrics), the central frequency of the ultrasonic transducer was $1 \mathrm{MHz}$, the bandwidth was 0.6 $\mathrm{MHz}$, the diameter was $1.9 \mathrm{~cm}$, and the focal length at 1 $\mathrm{MHz}$ was $2.5 \mathrm{~cm}$. For the second one (V384, Panametrics), the central frequency was $3.5 \mathrm{MHz}$, the bandwidth was 2.5 $\mathrm{MHz}$, the diameter was $0.64 \mathrm{~cm}$, and the focal length at 3.5 $\mathrm{MHz}$ was $1.8 \mathrm{~cm}$. Unless otherwise stated, the first ultrasonic transducer was used to obtained the results presented here.

In our scanning microwave-induced thermoacoustic tomography, the ultrasonic transducer measured the time-ofarrival signals of the thermoacoustic waves. The distance between the thermoacoustic source and the transducer was calculated by multiplying the time of arrival with the speed of sound in the medium. Therefore, a time-domain signal was converted into a one-dimensional image along the acoustic axis ( $z$ axis), which is similar to an ultrasonic A-scan image. Scanning the sample along the $x$ or $y$ axis and combining the multiple one-dimensional images yielded a two-dimensional cross-sectional image of the sample in the $x-z$ or $y-z$ plane.

\section{RESULTS AND DISCUSSION}

The generation of thermoacoustic waves by deposition of microwave energy can be described by the following differential equation:

$$
\left(\nabla^{2}-\frac{1}{v_{s}^{2}} \frac{\partial^{2}}{\partial t^{2}}\right) p(\mathbf{r}, t)=-\frac{\beta}{C_{p}} \frac{\partial H}{\partial t},
$$

where $p(\mathbf{r}, t)$ is the thermoacoustic pressure at position $\mathbf{r}$ and time $t, v_{s}$ is the speed of sound, $\beta$ is the isobaric volume expansion coefficient, $C_{p}$ is the heat capacity, and $H$ is the heating function describing the microwave-energy deposition in the sample per unit volume per unit time. Thermalconfinement condition is assumed, where the acoustic transit time across the acoustic source is less than the heat conduction time. The solution of the three-dimensional wave equation under the zero-initial-value conditions $p(0, \mathbf{r})=0$ and $(\partial / \partial t) p(0, \mathbf{r})=0$ can be expressed as an integral:

$$
p(\mathbf{r}, t)=\frac{\beta}{4 \pi C_{p}} \iiint \frac{1}{\left|\mathbf{r}-\mathbf{r}^{\prime}\right|} \frac{\partial H\left(\mathbf{r}^{\prime}, t^{\prime}\right)}{\partial t^{\prime}} d \mathbf{r}^{\prime} .
$$

The integral is calculated inside a sphere with a radius of $v_{s} t$ centered at $\mathbf{r}$, and $\mathbf{r}^{\prime}$ is the position inside the sphere where 


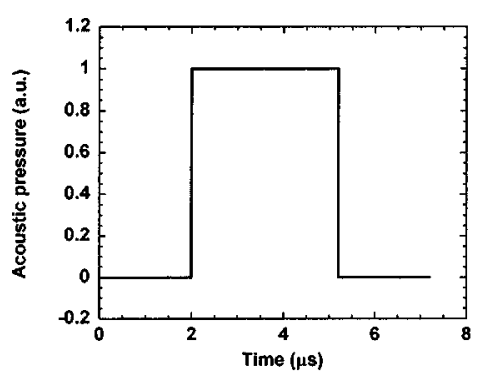

(a)

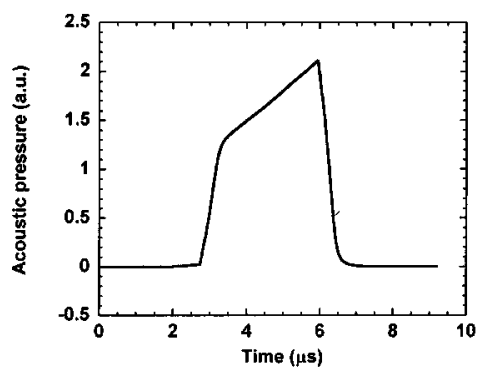

(c)

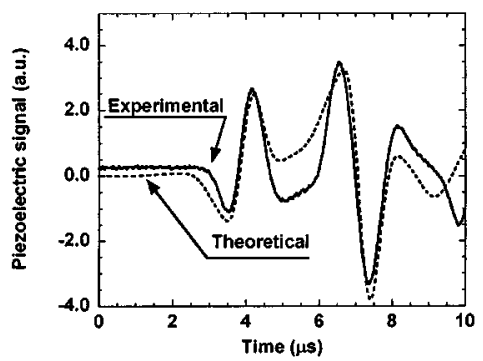

(e)

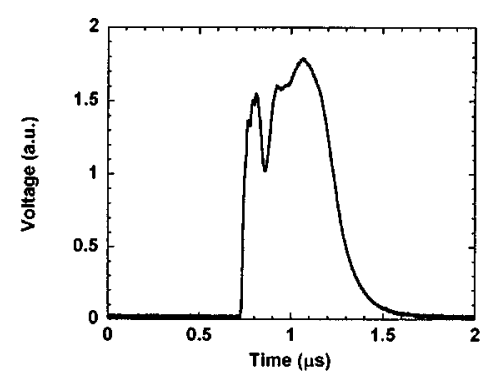

(b)

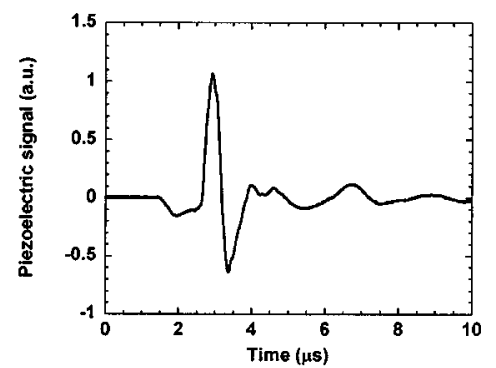

(d)
FIG. 2. Simulation of the piezo-electric signal in response to the microwave-induced thermoacoustic signal from a $4.8 \mathrm{~mm}$ thick gel slab. (a) Thermoacoustic signal in a slab induced by an ideal microwave impulse; (b) Temporal profile of the microwave pulses used in the experiment; (c) Thermoacoustic signal in a slab induced by the microwave pulses used in the experiment; (d) Piezo-electric impulse response of the ultrasonic transducer; (e) Experimental and simulated piezo-electric outputs of the ultrasonic transducer in response to the thermoacoustic signals. microwave is absorbed and acoustic signal is generated. In the integration, the heating function is not taken at time $t$ but at an earlier time $t^{\prime}=t-\left|\mathbf{r}-\mathbf{r}^{\prime}\right| / v_{s}$; therefore, the integration function is also called retarded potential. ${ }^{37}$ Analytic solutions can be obtained for simple geometric structures such as an infinite layer, a sphere, and a cylinder under delta heating, where the heating function is a delta function in time. ${ }^{38}$

For a slab with a thickness $d$ under delta heating, the impulse-response pressure is

$$
p_{1}(z, t)=\frac{\beta v_{s}^{2}}{2 C_{p}} u\left(z-v_{s} t\right),
$$

where $u\left(z-v_{s} t\right)$ is defined as a function that is unity when $0 \leqslant\left(z-v_{s} t\right) \leqslant d$ and zero otherwise. The impulse response is a traveling square wave as shown in Fig. 2(a) for a $4.8 \mathrm{~mm}$ gel slab if the microwave attenuation across the slab is negligible. Because the propagation speed of electromagnetic wave is much greater than the speed of sound, the sample volume illuminated by microwave pulses radiates acoustic waves simultaneously.

The heating function in one-dimensional lossy media may be expressed as $H(z, t)=\alpha e^{-\alpha z} s(t)$, where $\alpha$ is the micro- wave absorption coefficient, and $s(t)$ is the temporal profile of the microwave pulse. Figure 2(b) shows the temporal profile of the microwave pulses used in our experiment.

The thermoacoustic pressure induced by the microwave pulses can be derived by the following convolution:

$$
p(z, t)=\int p_{1}(z, \tau) H(z, t-\tau) d \tau
$$

Figure 2(c) illustrates the thermoacoustic pressure at the ultrasonic transducer generated from the slab, which was obtained by convolving the two temporal waveforms in Figs. 2(a) and 2(b) by use of Eq. (6).

For more general nonthermal-confined cases when heat transfer in the medium cannot be neglected, the following heat conduction equation must be taken into account in the pressure calculation:

$$
\rho C_{p} \frac{\partial T(\mathbf{r}, t)}{\partial t}=k \nabla^{2} T(\mathbf{r}, t)+H(\mathbf{r}, t)
$$

where $\rho$ is the density of the medium, $k$ is the thermal con- 
ductivity, and $T(\mathbf{r}, t)$ is the temperature distribution in the microwave-illuminated space. The thermoacoustic pressure is:

$$
\begin{aligned}
p(\mathbf{r}, t)= & \frac{\beta}{4 \pi C_{p}} \iiint \frac{1}{\left|\mathbf{r}-\mathbf{r}^{\prime}\right|}\left[\frac{\partial H\left(\mathbf{r}^{\prime}, t^{\prime}\right)}{\partial t^{\prime}}\right. \\
& \left.+k \nabla^{2} \frac{\partial T\left(\mathbf{r}^{\prime}, t\right)}{\partial t^{\prime}}\right] d \mathbf{r}^{\prime} .
\end{aligned}
$$

The integration involves the heat conducted from the surrounding medium besides the heat due to the absorbed microwave energy.

The experimentally measured piezo-electric impulse response $q(t)$ of the ultrasonic transducer is shown in Fig. 2(d). The piezo-electric output of the ultrasonic transducer in response to thermoacoustic pressure can be calculated by the following convolution between the thermoacoustic pressure at the transducer and the impulse response of the transducer:

$$
P_{0}(z, t)=\int p(z, \tau) q(\tau-t) d \tau .
$$

The piezo-electric signal from a $4.8 \mathrm{~mm}$ slab was obtained by the convolution of the two waveforms in Figs. 2(c) and 2(d) with Eq. (9) and is plotted in Fig. 2(e) as a dashed line.

The experimental piezo-electric signal from a $4.8 \mathrm{~mm}$ gel slab is plotted in Fig. 2(e) as a solid line for comparison, where the slight offset was caused by the dc drift of the amplifier in the experimental detection. There are two dipolar structures in each waveform in Fig. 2(e), and the signal between the dipolar structures is weak. The dipolar structures from the upper and lower surfaces of the slab have opposite polarities, which could not be explained by any existing theories in the literature. The polarity, the width of each dipole, and the distance between the two dipolar structures are in good agreement between the results of our theory and experiment. Because the piezo-electric signal of the ultrasonic transducer can be simulated by the two convolutions as shown above, the dipole width is related to the width of the microwave pulses and the width of the impulse response of the ultrasonic transducer, which were $0.5 \mu \mathrm{s}$ and $1.7 \mu \mathrm{s}$, respectively. The time intervals between the zero-crossing points of the two dipolar structures in the two waveforms are determined by the slab thickness and are equal to the acoustic transit time $3.2 \mu$ s over the 4.8-mm-thick slab.

Dipolar structures were also observed in photoacoustics by lasers, where a Q-switched laser with a pulse width of $\sim 10 \mathrm{~ns}$ and a wide-band ultrasonic transducer were employed. Our detected dipolar structures resemble the wellknown dipolar structures that originate from small spherical or cylindrical objects excited by laser pulses ${ }^{39}$ or from acoustic reflection at soft acoustic interfaces. ${ }^{40}$ However, our detected dipolar structures in the thermoacoustic signals in slabs resulted from the limited bandwidth of the ultrasonic transducers.

This phenomenon can also be explained in the frequency domain, where the ultrasonic transducer acts as a bandpass filter. A frequency spectrum of the ultrasonic transducer is

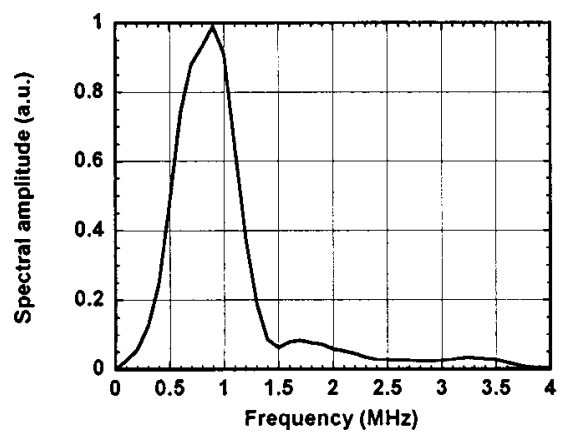

(a)

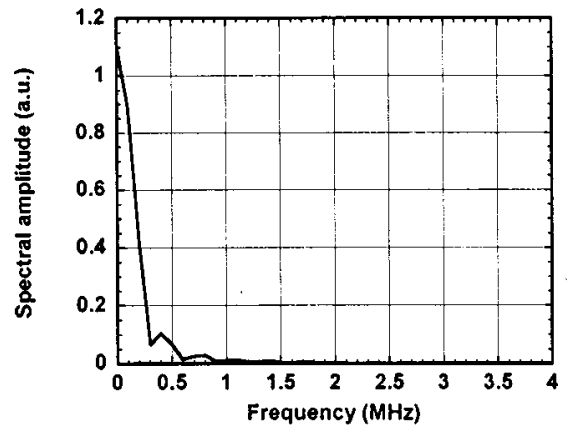

(b)

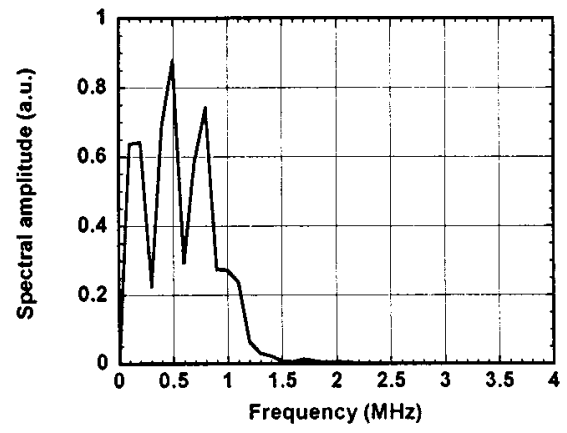

(c)

FIG. 3. Frequency-domain analysis of the microwave-induced thermoacoustic signals. (a) Spectrum of the $1 \mathrm{MHz}$ ultrasonic transducer; (b) Spectrum of the microwave-induced thermoacoustic signal; (c) Spectrum of the piezoelectric signal, which was the filtered microwave-induced thermoacoustic signal.

shown in Fig. 3(a). The temporal profile of the thermoacoustic pressure varies sharply near the slab boundaries and slowly inside the slab as shown in Fig. 2(c). The corresponding spectrum is peaked at dc as shown in Fig. 3(b). The filtered spectrum is peaked near $0.5 \mathrm{MHz}$ as shown in Fig. $3(\mathrm{c})$, where the dc is rejected and the low-frequency components are attenuated significantly. In other words, the ultrasonic transducer cannot respond efficiently to the thermoacoustic waves emitted between the sample boundaries, which have a lower-frequency spectrum. Therefore, the observed piezo-electric signal between the two sample boundaries is low. 
This model includes only the key concept. Scattering and diffraction of microwaves becomes certainly important if strictly quantitative modeling is performed. The detected thermoacoustic signals are also related to other factors including microwave attenuation, conversion efficiency from microwave energy to acoustic energy, acoustic scattering and diffraction, and acoustic attenuation.

A tissue sample with a fat-muscle-fat structure as shown in Fig. 4(a) was placed on the plastic stand inside the container as shown in Fig. 1. Figure 4(b) shows a 2D image of the sample obtained with the scanning thermoacoustic tomography technique. Thermoacoustic signals were acquired in the time domain while the fat-muscle-fat sample was scanned horizontally along the $y$ axis with a step size of 1 $\mathrm{mm}$. The 2D image of the sample was formed by combining these temporal waveforms taken successively at the scanning stops along the $y$ axis. Each vertical line in this 2D image was from a temporal waveform. The muscle inside the fat is clearly visible with a good contrast. The fat-tissue interface to the left of the muscle is also visible, which was possibly caused by the slight difference in the microwave properties between the two fat sections.

Figure 4(c) illustrates a time-domain waveform that was measured above the center of the sample at $y$ equal to 54 $\mathrm{mm}$. The strongest dipole near $27 \mu$ s was from the bottom boundary of the sample where microwave experienced the least attenuation, whereas the weakest dipole near $14 \mu \mathrm{s}$ was from the top boundary of the sample where microwave experienced the most attenuation. The two dipolar structures corresponding to the boundaries of the muscle layer are also clearly distinguishable. The time intervals between the adjacent dipolar structures agree with the thickness values of the tissue layers very well. However, the vertical boundaries of the muscle slab are not visible in the image because the thermoacoustic waves from these boundaries propagate perpendicularly to the acoustic axis of the ultrasonic transducer and therefore cannot be received by the transducer.

The axial resolution along the acoustic axis ( $z$ axis) is determined by the width of the thermoacoustic dipolar structures, which is related to the width of the microwave pulse and the width of the impulse response of the transducer (the inverse of the bandwidth of the ultrasonic detector). When the $1 \mathrm{MHz}$ ultrasonic transducer was used, the width of the thermoacoustic signal was estimated to be $2.2 \mu$ s, which was the sum of the width of the microwave pulses $(0.5 \mu \mathrm{s})$ and the width of the impulse response of the transducer $(1.7 \mu \mathrm{s})$. Because the speed of sound in tissue is $\sim 1.5 \mathrm{~mm} / \mu \mathrm{s}$, the corresponding axial resolution should be approximately 3.3 $\mathrm{mm}$ along the $z$ axis. For the $3.5 \mathrm{MHz}$ ultrasonic transducer, the axial resolution should be improved to $1.4 \mathrm{~mm}$ theoretically.

Figure 5 shows the thermoacoustic signals from slab samples of various thickness values measured by the $1 \mathrm{MHz}$ ultrasonic transducer. The slab samples were made of $5 \%$ gelatin and $5 \% \mathrm{NaCl}$, where $\mathrm{NaCl}$ controlled the microwave absorption. As the thickness of the samples decreased, the temporal distance between the adjacent dipolar structures corresponding to the two boundaries of the slabs decreased

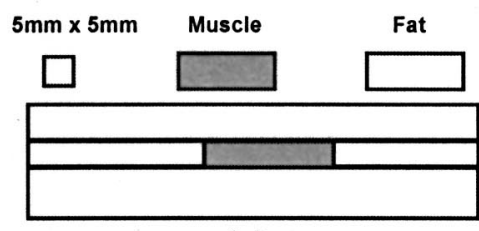

(a)

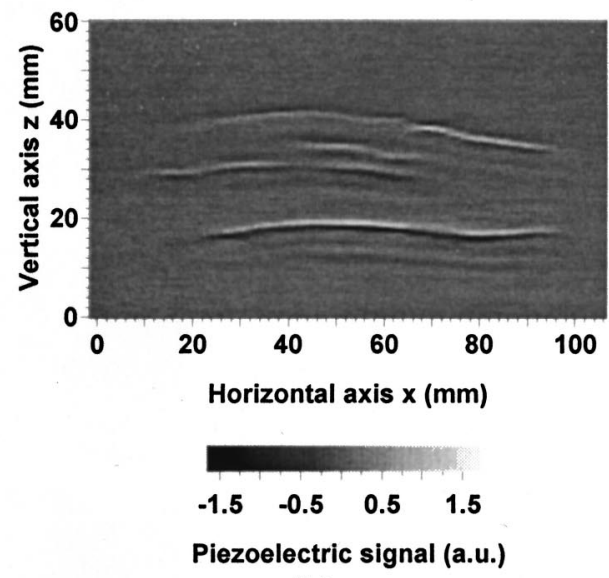

(b)

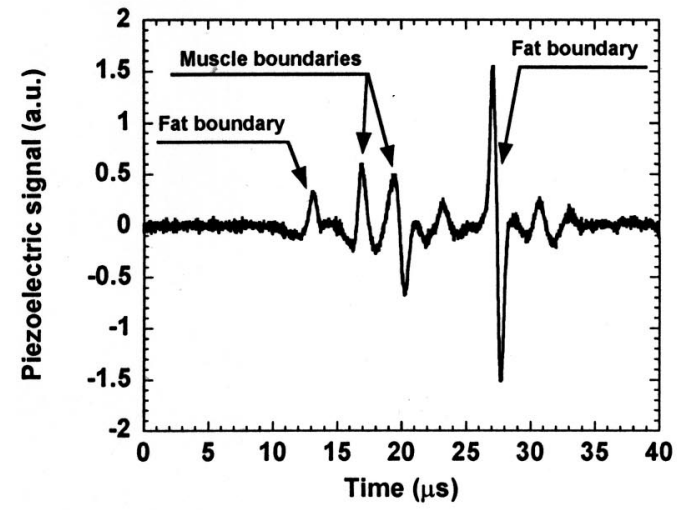

(c)

FIG. 4. (a) Cross section of a fat-muscle-fat sample on the $y-z$ plane; (b) Two-dimensional image of the $y-z$ cross section of the sample obtained by scanning thermoacoustic tomography; (c) Temporal microwave-induced thermoacoustic signal along the vertical center line of the sample. The 1 $\mathrm{MHz}$ ultrasonic transducer was used in this experiment.

as well. The two dipolar structures became barely distinguishable when the thickness was reduced to $3.8 \mathrm{~mm}$ and completely inseparable when the thickness was reduced to 2 $\mathrm{mm}$. Therefore, the experimentally measured axial resolution was $\sim 3.8 \mathrm{~mm}$, close to the above calculated resolution of 3.3 $\mathrm{mm}$ based on the dipole width. The discrepancy was caused by the long tail of the dipolar structures. The relative variation in intensity between the two dipolar structures was caused by microwave attenuation in the slabs.

Similarly, Fig. 6 shows the thermoacoustic signals from slab samples of various thickness values measured by the 3.5 


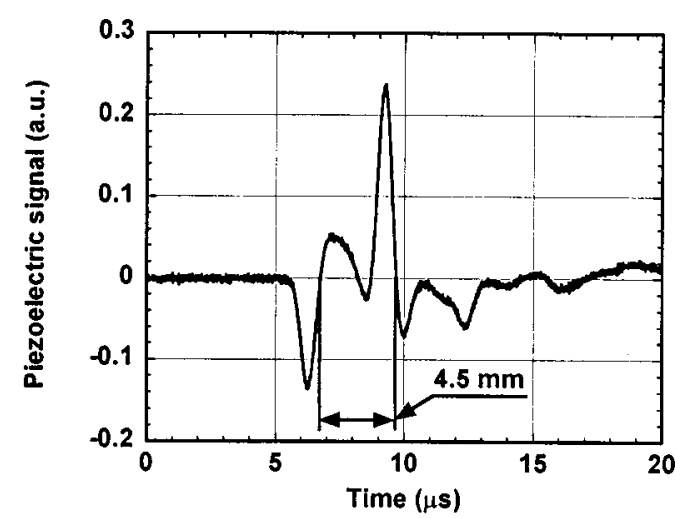

(a)

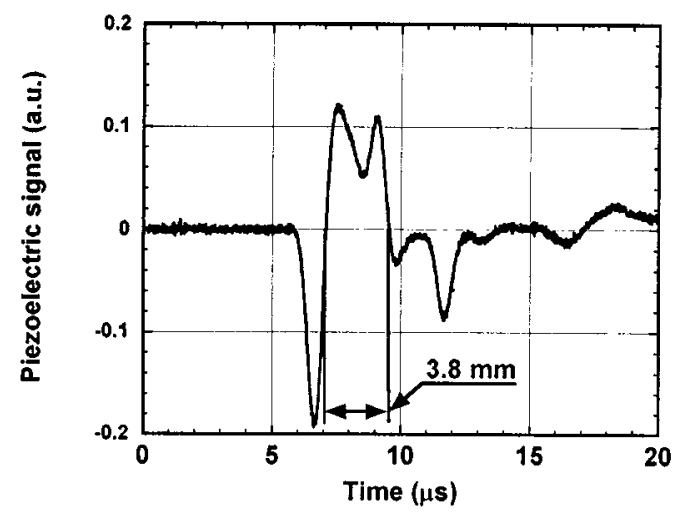

(b)

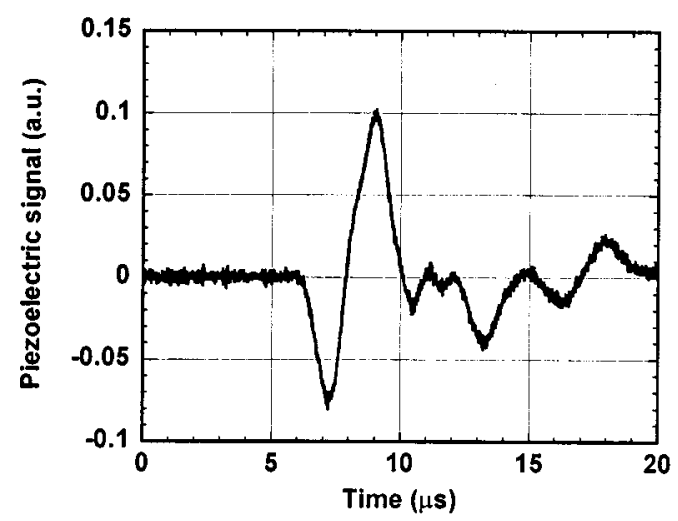

(c)

FIG. 5. Microwave-induced thermoacoustic signals in gel slabs of various thickness values: (a) $4.5 \mathrm{~mm}$, (b) $3.8 \mathrm{~mm}$, and (c) $2 \mathrm{~mm}$. The $1 \mathrm{MHz}$ ultrasonic transducer was used.

$\mathrm{MHz}$ ultrasonic transducer. The experimentally measured axial resolution was $\sim 1.9 \mathrm{~mm}$, close to the above calculated resolution of $1.4 \mathrm{~mm}$ based on the dipole width. As expected from the theoretical consideration, the wider-bandwidth transducer produced better axial resolution. Potentially, shorter microwave pulses and deconvolution may be used to improve the axial resolution further.

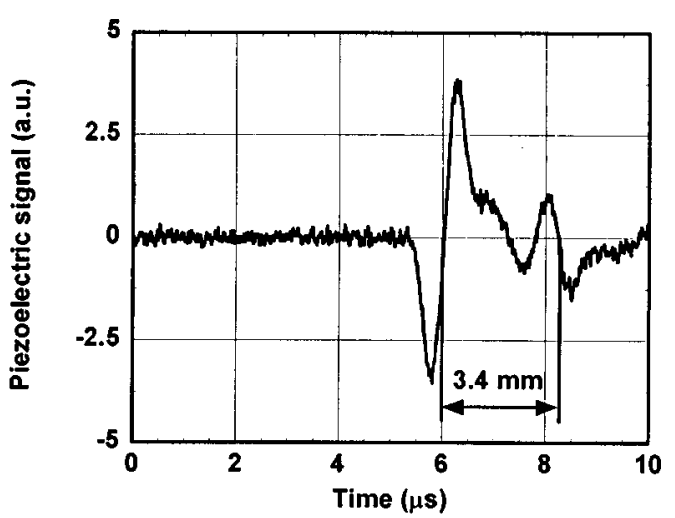

(a)

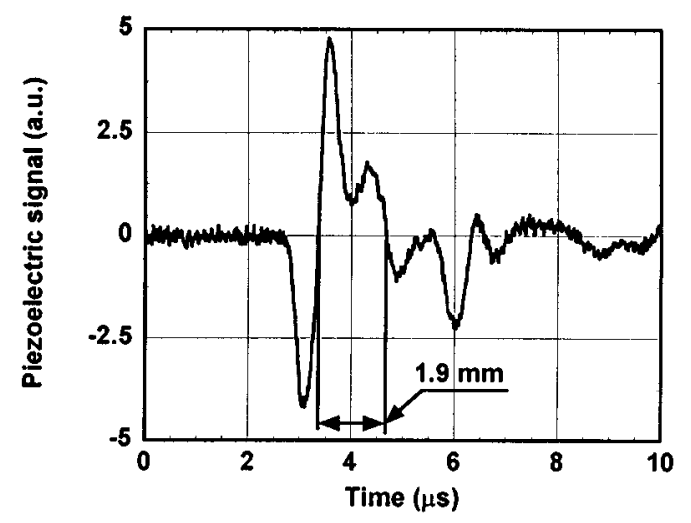

(b)

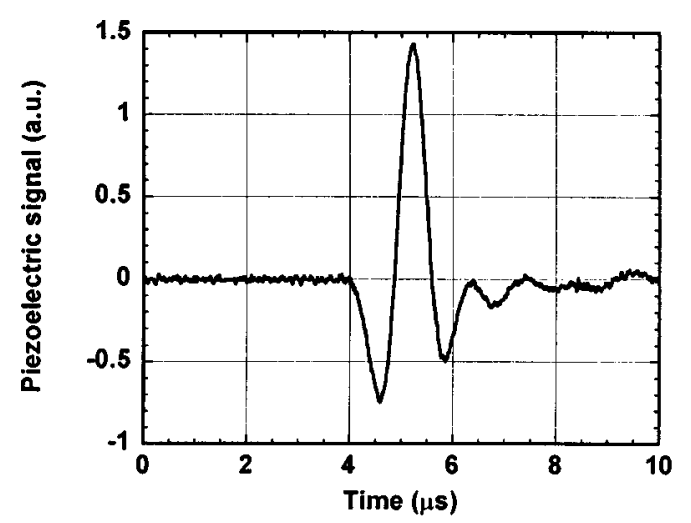

(c)

FIG. 6. Microwave-induced thermoacoustic signals in gel slabs of various thickness values: (a) $3.4 \mathrm{~mm}$, (b) $1.9 \mathrm{~mm}$, and (c) $1 \mathrm{~mm}$. The $3.5 \mathrm{MHz}$ ultrasonic transducer was used.

The lateral resolution is determined by the numerical aperture of the ultrasonic transducer. The ultrasonic transducer responds to the thermoacoustic signals along its acoustic axis. The detected source area is related to the numerical aperture of the ultrasonic transducer and the distance between the thermoacoustic source and the ultrasonic transducer. The minimum detected source area is at the focal 


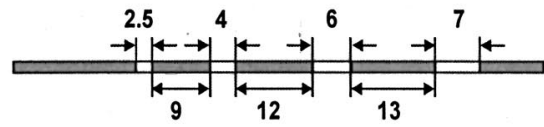

(a)
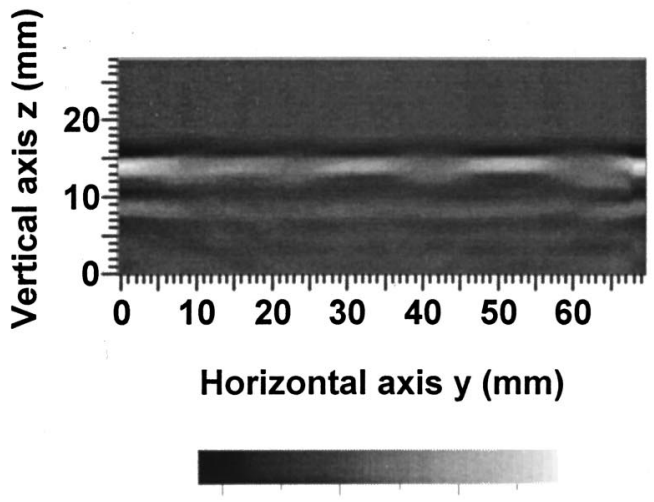

$\begin{array}{lll}-0.02 & 0.00 & 0.02\end{array}$

Piezoelectric signal (a.u.)

(b)

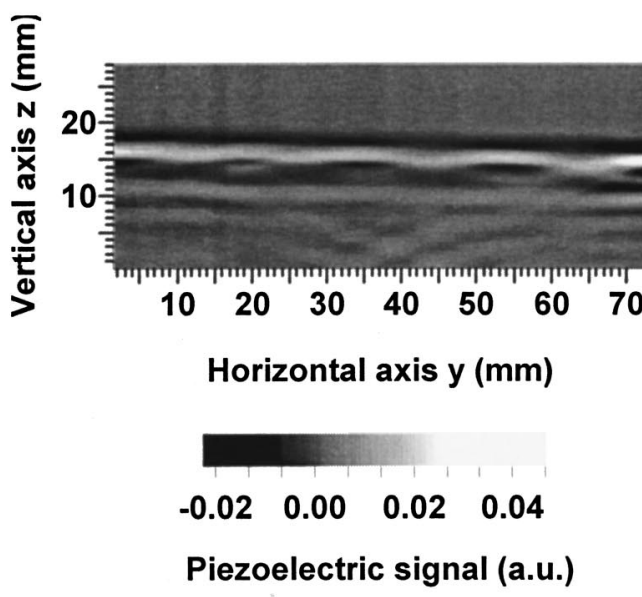

(c)

FIG. 7. Two-dimensional tomographic images of a linear array of gel slabs obtained by scanning thermoacoustic tomography. (a) $y-z$ cross section of the sample (units in $\mathrm{mm}$ ); (b) Image of the $y-z$ cross section when the sample was placed at the focal plane of the ultrasonic transducer; (c) Image of the $y-z$ cross section when the sample was placed far from the focal plane of the ultrasonic transducer.

plane of the ultrasonic transducer. Therefore, a better lateral resolution is expected when the sample is located within the focal column. We examined the lateral resolution with the 1 $\mathrm{MHz}$ ultrasonic transducer. The ultrasonic transducer has a 3 $\mathrm{dB}$ focal diameter of $2.1 \mathrm{~mm}$ and a focal zone of $17.6 \mathrm{~mm}$ along the acoustic axis. Several pieces of rectangular gel slabs were arranged linearly along the $y$ direction as shown in Fig. 7(a). The $y-z$ cross section was imaged with a step size of $1 \mathrm{~mm}$ when the sample was scanned along the $y$ axis. Figure 7(b) shows the two-dimensional image when the sample was on the focal plane of the ultrasonic transducer. The bright upper band near $z=14 \mathrm{~mm}$ is the primary image from the thermoacoustic waves propagating directly upward toward the ultrasonic transducer, whereas the dark lower band near $z=9 \mathrm{~mm}$ is the "ghost" image caused by acoustic reflection from the plastic stand. The gaps of greater than 4 $\mathrm{mm}$ can be easily recognized. The gap of $2.5 \mathrm{~mm}$ can be barely identified, which defines the lateral resolution and is comparable with the focal diameter of the ultrasonic transducer.

The measured thermoacoustic signal is a convolution between the thermoacoustic signal in the sample and the detection-sensitivity distribution of the ultrasonic transducer over the detected area. The convolution reduces the lateral resolution, which is worsened when the ultrasonic transducer is out of focus. Figure 6(c) was acquired when the ultrasonic transducer was deliberately moved far away from the sample so as to create defocusing. The gaps are not distinguishable in the $2 \mathrm{D}$ image due to reduced resolution.

\section{CONCLUSIONS}

The simulation of the piezo-electric signal, by convolving the thermoacoustic signal of delta heating with the microwave-pulse waveform and then with the impulse response of the ultrasonic transducer, can be used to explain the bipolar experimental data. Our studies show that scanning microwave-induced thermoacoustic tomography is a promising imaging tool for biological tissue. The boundaries of different tissue constituents can be imaged clearly. The width of the microwave pulses and the bandwidth of the ultrasonic transducer determine the axial resolution along the acoustic axis of the ultrasonic transducer, which is $\sim 1.9 \mathrm{~mm}$ under our current experimental conditions. The axial resolution can be improved by employing broader-band ultrasonic transducers and shorter microwave pulses. The lateral resolution is determined by the numerical aperture of ultrasonic transducers and is $\sim 2.5 \mathrm{~mm}$ in our current setup.

\section{ACKNOWLEDGMENTS}

Thanks to X. Zhao and G. Yao for their assistance in electronics and computer programming, respectively. This project was sponsored in part by the National Institutes of Health Grants Nos. R29 CA68562, R01 CA71980, and R21 CA83760 and by the National Science Foundation Grant no. BES-9734491.

a) To whom correspondence should be addressed: Tel: (979) 847-9040; Fax: (979) 845-4450; electronic mail: lwang@tamu.edu; URL: http:// oilab.tamu.edu

${ }^{1}$ L. E. Larsen and J. H. Jacobi, Eds., Medical Applications of Microwave Imaging (IEEE Press, Piscataway, NJ, 1986).

${ }^{2}$ J. C. Lin, "Frequency optimization for microwave imaging of biological tissues,' Proc. IEEE 73, 374-375 (1985).

${ }^{3}$ S. Caorsi, A. Frattoni, G. L. Gragnani, E. Nortino, and M. Pastorino, "Numerical algorithm for dielectric-permittivity microwave imaging of inhomogeneous biological bodies," Med. Biol. Eng. Comput. 29, NS37-44 (1991).

${ }^{4}$ M. S. Hawley, A. Broquetas, L. Jofre, J. C. Bolomey, and G. Gaboriaud, "Microwave imaging of tissue blood content changes,', J. Biomed. Eng. 13, 197-202 (1991). 
${ }^{5}$ P. M. Meaney, K. D. Paulsen, and J. T. Chang, "Near-field microwave imaging of biologically-based materials using a monopole transceiver system," IEEE Trans. Microwave Theory Tech. 46, 31-45 (1998).

${ }^{6}$ J. C. Lin, "'On microwave-induced hearing sensation," IRE Trans. Microwave Theory Tech. MTT-25, 605-613 (1977).

${ }^{7} \mathrm{~F}$. Caspers and J. Conway, "Measurement of power density in a lossy material by means of electromagnetically-induced acoustic signals for noninvasive determination of spatial thermal absorption in connection with pulsed hyperthermia," Proc. of the 12th European Microwave Conference, 565-568 (1982).

${ }^{8}$ J. L. Su and J. C. Lin, "Thermoelastic signatures of tissue phantom absorption and thermal expansion,"' IEEE Trans. Biomed. Eng. 43, 178182 (1987)

${ }^{9}$ J. S. K. Wan, "Microwaves and chemistry: The catalysis of an exciting marriage," Res. Chem. Intermed. 19, 147-158 (1993).

${ }^{10}$ T. Bowen, L. Nasoni, A. E. Pifer, and G. H. Sembrosk, "Some experimental results on the thermoacoustic imaging of soft tissue-equivalent phantoms," Ultrason. Symp. Proc. 2, 823-827 (1981).

${ }^{11}$ R. G. Olsen, "Generation of acoustic images from the absorption of pulsed microwave energy," in Acoustic Imaging, edited by J. P. Powers (Plenum, New York, 1982), pp. 53-59.

${ }^{12}$ R. G. Olsen and J. C. Lin, "Acoustic imaging of a model of a human hand using pulsed microwave irradiation," Bioelectromagnetics (N.Y.) 4, 397-400 (1983).

${ }^{13}$ J. C. Lin and K. H. Chan, "Microwave thermoelastic tissue imagingsystem design," IEEE Trans. Microwave Theory Tech. 32, 854-860 (1984).

${ }^{14}$ R. L. Nasoni, G. A. Evanoff, Jr., P. G. Halverson, and T. Bowen, "Thermoacoustic emission by deeply penetrating microwave radiation," Proc.IEEE Ultrason. Symp. 5, 633-637 (1984).

${ }^{15}$ K. H. Chan and J. C. Lin, "Microwave-induced thermoacoustic tissue imaging," Proc. Engineering in Medicine and Biology Society 10th Annual International Conference, 445-446 (1988).

${ }^{16}$ R. A. Kruger, D. R. Reinecke, and G. A. Kruger, "Thermoacoustic computed tomography-Technical considerations,' Med. Phys. 26, 18321837 (1999).

${ }^{17}$ R. A. Kruger, K. K. Kopecky, A. M. Aisen, D. R. Reinecke, G. A. Kruger, and W. L. Kiser, Jr., "Thermoacoustic CT with radio waves: a medical imaging paradigm," Radiology 211, 275-278 (1999).

${ }^{18}$ V. E. Gusev and A. A. Karabutov, Laser Optoacoustics (American Institute of Physics, New York, 1993).

${ }^{19}$ A. A. Oraevsky, S. L. Jacques, R. O. Esenaliev, and F. K. Tittel, "'Laserbased optoacoustic imaging in biological tissues," in Laser-Tissue Interaction $V$, edited by S. L. Jacques, Proc. Soc. Photo-Instrum. Eng. 2134A, 122-128 (1994).

${ }^{20}$ R. A. Kruger and P. Liu, "Photoacoustic ultrasound: Pulse production and detection of 0.5\% Liposyn," Med. Phys. 21, 1179-1184 (1994).

${ }^{21}$ A. A. Oraevsky, S. L. Jacques, R. O. Esenaliev, and F. K. Tittel, “'Direct measurement of laser fluence distribution and optoacoustic imaging in heterogeneous tissues," in Laser Interaction with Hard and Soft Tissue II, edited by H. J. Albrecht, G. P. Delacretaz, T. H. Meier, R. W. Steiner, L. O. Svaasand, and M. J. van Gemert, Proc. Soc. Photo-Instrum. Eng. 2323, 37-46 (1995).

${ }^{22}$ R. A. Kruger, P. Liu, Y. R. Fang, and C. R. Appledorn, "Photoacoustic ultrasound (PAUS) - reconstruction tomography," Med. Phys. 22, 16051609 (1995).

${ }^{23}$ A. A. Oraevsky, R. Esenaliev, F. K. Tittel, M. R. Ostermeyer, L.-H. Wang, and S. L. Jacques, "Laser opto-acoustic imaging of turbid media: determination of optical properties by comparison with diffusion theory and Monte Carlo simulation,' Proc. SPIE 2681, 277-284 (1996).

${ }^{24}$ A. A. Oraevsky, R. O. Esenaliev, and A. A. Karabutov, "Laser optoacoustic tomography of layered tissues: signal processing," in Optical Tomography and Spectroscopy of Tissue: Theory, Instrumentation, Model, and Human Studies II, edited by B. Chance and R. R. Alfano, Proc. Soc. Photo-Instrum. Eng. 2979, 59-70 (1997).

${ }^{25}$ R. O. Esenaliev, H. Alma, F. K. Tittel, and A. A. Oraevsky, "Axial resolution of laser opto-acoustic imaging: influence of acoustic attenuation and diffraction," in Laser-Tissue Interaction IX, edited by S. L. Jacques, Proc. Soc. Photo-Instrum. Eng. 3254, 294-306 (1998).

${ }^{26}$ C. G. A. Hoelen, F. F. M. Demul, R. Pongers, and A. Dekker, "'Threedimensional photoacoustic imaging of blood vessels in tissue," Opt. Lett. 23, 648-650 (1998).

${ }^{27}$ A. A. Oraevsky, V. A. Andreev, A. A. Karabutov, and R. O. Esenaliev, "Two-dimensional optoacoustic tomography: Transducer array and image reconstruction algorithm," in Laser-Tissue Interaction X: Photochemical, Photothermal, and Photomechanical, edited by S. L. Jacques, G. J. Mueller, A. Roggan, and D. H. Sliney, Proc. Soc. Photo-Instrum. Eng. 3601, 256-267 (1999).

${ }^{28}$ A. A. Karabutov, E. B. Savateeva, and A. A. Oraevsky, "Imaging of layered structures in biological tissues with optoacoustic front surface transducer," in Laser-Tissue Interaction X: Photochemical, Photothermal, and Photomechanical, edited by S. L. Jacques, G. J. Mueller, A. Roggan, and D. H. Sliney, Proc. Soc. Photo-Instrum. Eng. 3601, 284295 (1999).

${ }^{29}$ C. Gabriel, S. Gabriel, and E. Corthout, "The dielectric properties of biological tissues: I. literature survey,” Phys. Med. Biol. 41, 2231-2249 (1996).

${ }^{30} \mathrm{~S}$. Gabriel, R. W. Lau, and C. Gabriel, "The dielectric properties of biological tissues: II. Measurements in the frequency range $10 \mathrm{~Hz}$ to 20 GHz,' Phys. Med. Biol. 41, 2251-2269 (1996).

${ }^{31}$ S. Gabriel, R. W. Lau, and C. Gabriel, "The dielectric properties of biological tissues: III. Parametric models for the dielectric spectrum of tissues," Phys. Med. Biol. 41, 2271-2293 (1996).

${ }^{32}$ F. A. Duck, Physical Properties of Tissue: A Comprehensive Reference Book (Academic, London, 1990).

${ }^{33}$ W. Joines, R. Jirtle, M. Rafal, and D. Schaeffer, "Microwave power absorption differences between normal and malignant tissue," Int. J. Radiat. Oncol. Biol. Phys. 6, 681-687 (1980).

${ }^{34}$ S. Chaudhary, R. Mishra, A. Swarup, and J. Thomas, "Dielectric properties of normal human breast tissues at radiowave and microwave frequencies," Indian J. Biochem. Biophys. 21, 76-79 (1984).

${ }^{35}$ W. Joines, Y. Zhang, C. Li, and R. Jirtle, "The measured electrical properties of normal and malignant human tissues from 50-900 MHz," Med. Phys. 21, 547-550 (1994).

${ }^{36}$ L.-H. Wang, X. Zhao, H. Sun, and G. Ku, "Microwave-induced acoustic imaging of biological tissues," Rev. Sci. Instrum. 70, 3744-3748 (1999).

${ }^{37}$ I. N. Bronshtein and K. A. Semendyayev, Handbook of Mathematics (Van Nostrand Reinhold, New York, 1978).

${ }^{38}$ G. J. Diebold and T. Sun, "Properties of photoacoustic wave in one two and three dimensions," Acustica 80, 339-351 (1994)

${ }^{39}$ C. G. A. Hoelen, F. F. M. de Mul, and J. Greve, "Non-destructive photoacoustic subsurface tissue imaging: a feasibility study," Proc. SPIE 2628, 304-314 (1995)

${ }^{40}$ A. O. Oraevsky, S. L. Jacques, and F. K. Tittel, "Measurement of tissue optical properties by time-resolved detection of laser-induced transient stress,"’ Appl. Opt. 36, 402-415 (1997). 